\title{
Single Molecule Imaging and Manipulation
}

\author{
Martin Hegner ${ }^{\mathrm{b} *}$ and Andreas Engel ${ }^{\mathrm{a} *}$
}

\begin{abstract}
The atomic force microscope (AFM) and optical tweezers are tools that allow single biomolecules to be imaged and manipulated. Progress in instrumentation, sample preparation, and image acquisition conditions make novel applications of these tools possible. Biological membranes can be imaged in their native state at a lateral resolution of $0.4-1 \mathrm{~nm}$ and a vertical resolution of $0.1-0.2 \mathrm{~nm}$. Function-related conformational changes are resolved to a similar resolution, complementing atomic structure data acquired by other methods. The unique capability of the AFM to observe single proteins directly allows the interaction of proteins forming functional assemblies to be assessed. Single molecule force spectroscopy combined with single molecule imaging provides unprecedented possibilities to analyze intra- and intermolecular forces. Optical tweezers expand the range of measurable forces to those produced by molecular motors. Combined with fluorescence measurements, optical tools give insights into fundamental biological processes such as the molecular conversion of chemical into mechanical energy.
\end{abstract}

Keywords: Atomic force microscope · Membrane proteins · Molecular motors · Optical Tweezers · Single molecules

\section{Introduction}

Biomolecules can only be observed at work if they reside in their native environment. For soluble proteins this is a physiological buffer, while membrane proteins in addition need to be embedded in a lipid bilayer. The atomic force microscope (AFM) [1] is the only instrument that provides subnanometer spatial resolution and can be operated in solution. Progress has been achieved in several laboratories by optimizing sample preparation [2-5] and image ac-

${ }^{\star}$ Correspondence: aprof. A. Enge

M.E. Müller Institute for Structural Biology

Biozentrum, University of Basel

NCCR Nanoscale Science

Klingelbergstrasse 50-70

$\mathrm{CH}-4056$ Basel

Tel.: + 41612672262

Fax: + 41612672109

E-Mail: Andreas.Engel@unibas.ch

bPD Dr. M. Hegner

Institut für Physik der Universität Basel

NCCR Nanoscale Science

Klingelbergstrasse 82

$\mathrm{CH}-4056$ Basel

Tel.: +41612673761

Fax: +41612673784

E-Mail: Martin.Hegner@unibas.ch quisition [6][7] methods, and by continuous developments of the instrumentation [8-11].

Topographs (maps of the surface topography) of biomolecules acquired with the AFM reveal the object in its most native state. The high signal-to-noise $(\mathrm{S} / \mathrm{N})$ ratio provided by this instrument allows submolecular features of single biomolecules to be discerned. Structural changes at their surfaces can be detected with a sufficient time resolution to monitor conformational changes involved in biological processes. In addition, the AFM stylus is a nanotool that allows single molecules to be manipulated. Supramolecular structures and single biomolecules can be dissected, using the sharp tip as nanoscalpel, or biomolecules can be attached to the stylus and unfolded while the stylus is retracted. The forces measured during this process give novel information on the nature of molecular interaction and the forces that stabilize the building blocks of live. While the AFM measures forces between $20 \mathrm{pN}$ and 2000 $\mathrm{pN}\left(1 \mathrm{pN}=10^{-12} \mathrm{~N}\right)$, optical tweezers are even more sensitive, allowing the detection of forces smaller than $0.3 \mathrm{pN}$. Using such optical tools, forces generated by single molecular motors can be directly measured, and the nanomechanics of single biomolecules investigated.

\section{Instrumentation}

\section{The Atomic Force Microscope}

A topograph is recorded by raster scanning the sample below the stylus that is attached to a flexible cantilever while a servo displaces the sample vertically to keep the cantilever deflection constant. An optical system resolves cantilever deflections of $0.1 \mathrm{~nm}$, which corresponds to a force difference of typically $10-50 \mathrm{pN}$. With modern instruments stable contact mode operation (see Fig. 1) is possible at forces of some $50 \mathrm{pN}$, provided the sample is in an aqueous solution. Various ways to exploit the deflection signal yield quite different types of images, as illustrated and explained in Fig. 1A. The possibility to raster scan and manipulate biological macromolecules under physiological conditions is a major advantage of the AFM. Fig. 1B displays the experimental details of an AFM, which allows scanning a sample in buffer solution, and exhibits an efficient system for changing the buffer.

The sensitivity of the cantilever deflection detector has promoted force measurements with the AFM. Unfolding forces of biomolecules that are tethered to substrate and tip can be monitored while retracting the tip. To record such force-distance curves, the tip is approached vertically to- 
wards the sample until a molecule is attached to the tip, and subsequently retracted ('fly-fishing'). Acquisition of high-resolution images before and after 'unzipping' a biomolecule allows the damage produced to be directly visualized. Only specialized AFMs can resolve forces below $20 \mathrm{pN}$, a limitation preventing the direct monitoring of molecular motors, whose forces are in the range of $1-10 \mathrm{pN}$.

\section{The Optical Tweezers}

Optical tweezers (OTs) measure forces as small as $0.3 \mathrm{pN}$. To this end, a laser beam is focused to a diffraction-limited spot using a microscope objective. The intense light gradient near the focal region can achieve stable three-dimensional trapping of dielectric objects, varying in size from a few tens of nanometers up to tens of micrometers (Fig. 2A). As result of its high force sensitivity the OTs are considered as the technique of choice for the investigation of biomechanical forces. A single molecule can be attached to a handle such as a small dielectric sphere, and its mechanical properties can be studied (Fig. 2B). Fig. 3A illustrates that the force regimes in which

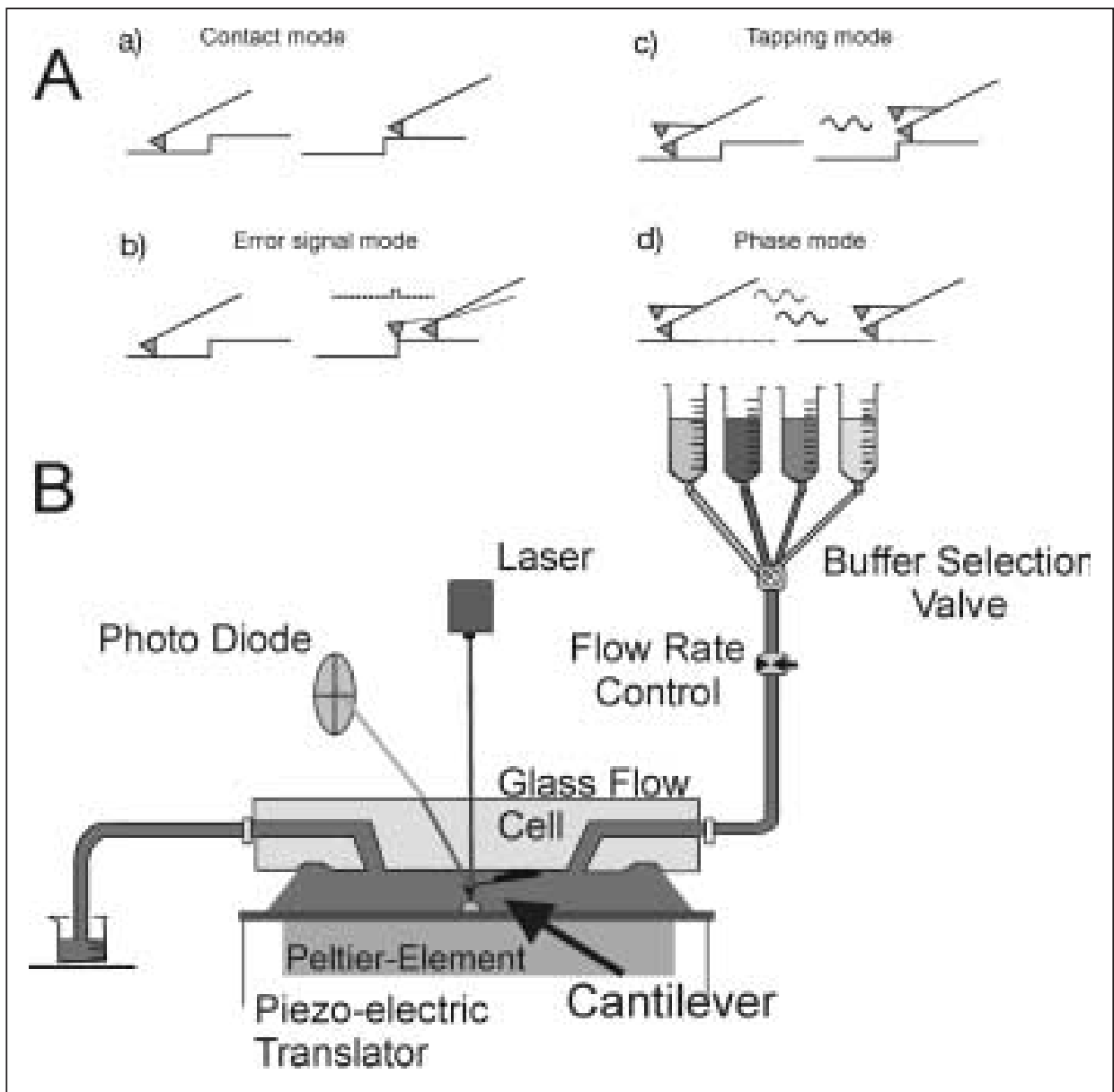

Fig. 1. AFM Instrumentation and modes of operation. A) Imaging modes: In the contact mode (a) the servo system moves up over elevations and down over depressions while the sample is raster scanned below the stylus. To react the servo needs to a difference signal from keep the cantilever deflection constant at sharp edges (b). This error signal provides an image that reveals the edges of the surface topography. Approaching the sample with an oscillating cantilever results in tapping of the sample by the stylus (c). This reduces the oscillation amplitude providing the signal to activate the servo. Because the tip-sample contact is disrupted periodically, the friction forces are eliminated. The phase difference between the measured oscillation (solid wave) and the oscillation driving the cantilever depends on the mechanical properties of the sample (d). This phase signal thus produces a sensitive material contrast.

B) Key elements of an AFM are the cantilever with a pyramidal stylus that touches the sample, an optical lever consisting of a laser and a photo diode to measure the cantilever's deflection, a piezo-electric translator to displace the sample in $\mathrm{x}, \mathrm{y}, \mathrm{z}$, and a computer to control these movements and store the surface contours. The instrument is working in buffer solution under ambient condition. The springboard type cantilever (dimensions $\sim 200 \times 20 \times 0.5 \mu \mathrm{m}$, spring constant $0.1-0.01 \mathrm{~N} / \mathrm{m}$ ) is deflected upwards when the tip is pushed towards the sample surface (repulsive forces) or downwards when the tip is retracted from the sample surface (attractive forces). Different liquids can be injected and a Peltier element allows precise adjustment of the temperature. the OTs and AFM can be used overlap conveniently. OTs are preferably applied in experiments on molecular motors, entropic elasticity of molecules, and conformational folding of proteins, while rupture of bonds and molecular interactions are mainly investigated with the AFM. While some technical details of OTs instruments are given below, a more detailed description of OTs is given in [11].

\section{Origin of Optical Forces}

As demonstrated in 1970 by Ashkin light can be used to trap and accelerate dielectric micron-sized particles [12]. For this experiment, a stable optical potential well was formed using two slightly divergent counter-propagating laser beams. This pioneering study established the groundwork for the OTs technique [13]. At the diffraction-limited focus of a laser beam, not only dielectric particles spheres can be trapped but also biological organisms such as cells, virus, or bacteria [14-16]. However, optical forces are very small, since $100 \mathrm{~mW}$ of power focused to a spot of $1 \mu \mathrm{m}$ diameter $\left(\approx 10^{7} \mathrm{~W} / \mathrm{cm}^{2}\right)$ produces forces of only a few tens of $\mathrm{pN}$ on a micron-sized particle (Fig. 2A). For biological applications, it is therefore imperative to choose a laser excitation, which (i) does not raise the temperature of the surrounding medium (i.e. water) and (ii) prevents biological damage. Near infrared excitation is best suited, but the wavelength region between 700 and $760 \mathrm{~nm}$ should be avoided [17][18].

\section{Experimental Details}

An optical trap for investigating biomolecules consists of (i) a beam expander to overfill the back aperture of the microscope lens, (ii) a high-quality microscope lens with a high NA to produce a steep spatial gradient, and (iii) a laser providing a wavelength in the near infrared. Some rather simple modifications of a commercial inverted microscope are sufficient to build an OTs apparatus [19]. When the requirements such as beam steering, high mechanical stability, proper spatial filtering of the laser, and preventing mode hopping of the laser have to be considered, it is best to build an OTs instrument on a conventional optical table with custom optics and electronics [16]. As a result of the difference in index of refraction between oil and water inside the chamber, oil-immersion microscope lenses are not suited for OTs. Fortunately, high numerical water-immersion lenses are now available commercially. Our OTs setup allows complementary biological information to be gathered (Fig. 2B). 

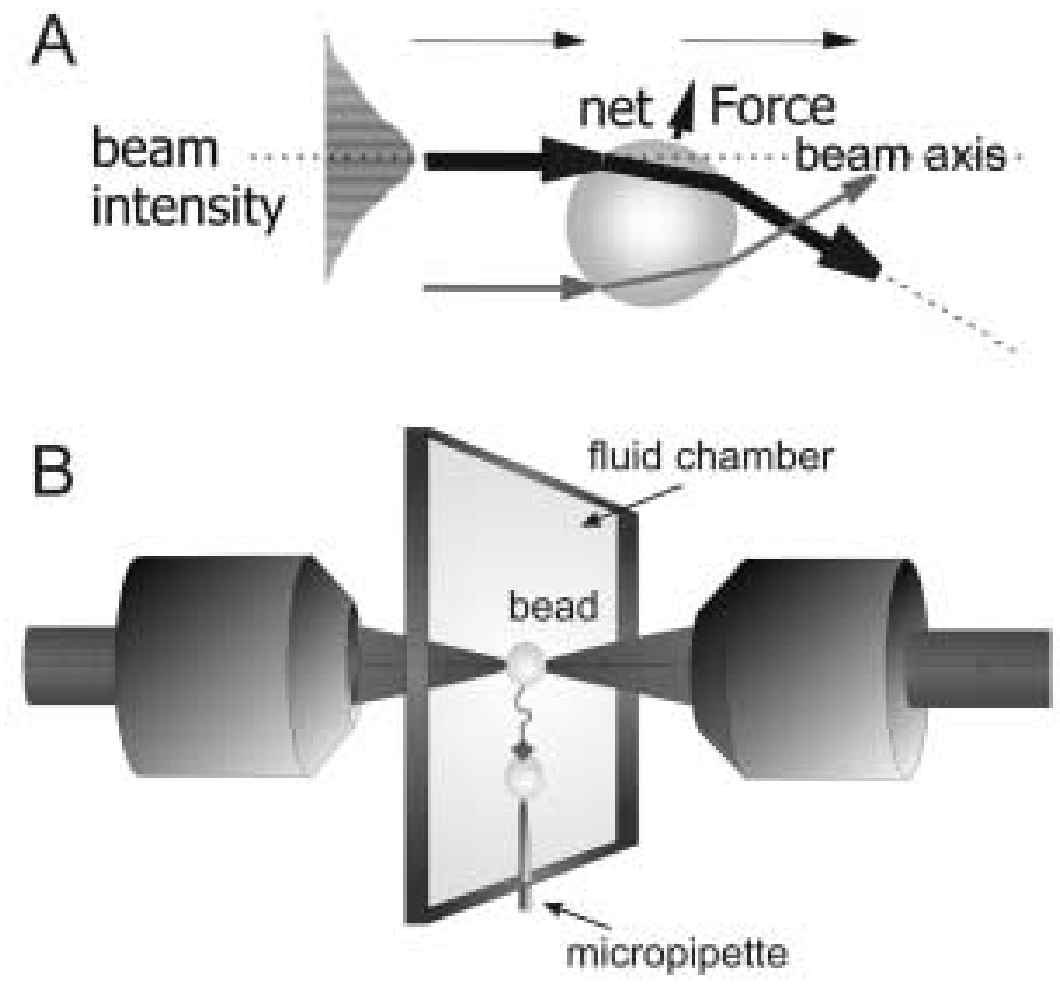

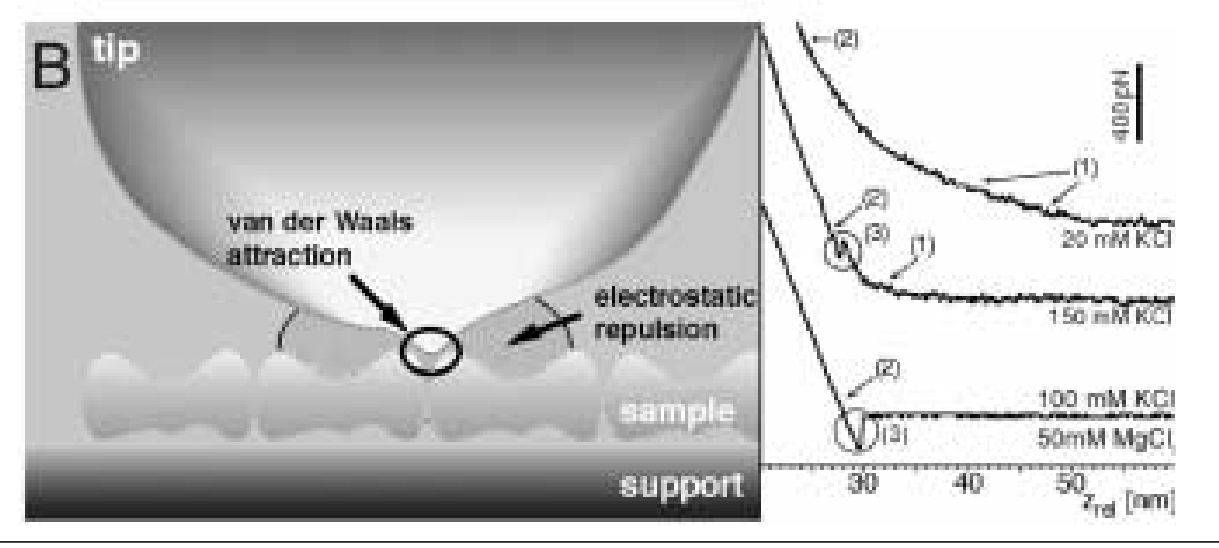

Fig. 2. Optical tweezers. A) Optical forces: A transparent dielectric micron-sized particle with an index of refraction larger than the surrounding medium is pushed towards the largest intensity of the light. All light rays are refracted when entering the particle. Due to the second Newton's law, the change in light momentum flux (the force) causes a reaction force on the particle. Center rays contain more photons than outer rays and exert therefore more force. The resulting net force is shown. B) Schematic representation of an OTs experiment. A microscope objective lens with a high $N A$ is used to focus the laser light to a diffraction-limited spot. At the focus, where the spatial gradient is maximal, particles such as beads can be trapped. Light is collected with a condenser lens, which converts angular deflections into transverse deflections that can be monitored on a position sensitive detector. A single molecule can be attached between the trapped bead and a bead on a micropipette through a receptor-ligand bridge. $\mathrm{Me}$ chanical properties of single molecules can be therefore investigated if the micropipette is placed onto a piezoelectric element. When the trapped bead experiences the force (arrow), it moves slightly away from its stable position. As in AFM, this leads to a deflection on the detector.
Fig. 3. Molecular forces. A) Overview on techniques that are applied to assess forces in biological systems in an aqueous environment. On the right a series of experiments in the various areas of motor proteins or molecular mechanics are shown. Some of the experiments like unfolding of individual proteins and dissociating (bio-molecular) bonds depend on the rate of the applied external force. B) DLVO Forces: Force-distance curves recorded on the extracellular purple membrane surface. The data was obtained for different electrolyte concentrations at constant $\mathrm{pH}$ (7.6). Force curves were recorded during the approach of sample and AFM tip. The dotted lines (....) represent force-distance curves expected for an experimental situation where electrostatic repulsion and van der Waals attration are balanced. Conditions: scan frequency $1.97 \mathrm{~Hz}$; scan range $50 \mathrm{~nm}(512$ pixel). Arrows (1) mark the onset of measurable electrostatic repulsion, whereas arrows (2) indicate the point of contact between tip and sample. 


\section{Imaging and Manipulation of Single Biomolecules with the AFM}

\section{Imaging}

To achieve high resolution, topographs are recorded in buffer solution. In a simplified model, electrostatic and van der Waals forces govern the tip-sample interactions in aqueous solutions. Hydrophilic surfaces are charged in water, leading to long-range electrostatic interactions. They can be attractive or repulsive, depending on the surface charges, which depend on the $\mathrm{pH}$. Screening the surface charges with electrolytes allows the electrostatic interactions to be controlled. Since the stylus (silicon nitride, $\mathrm{Si}_{3} \mathrm{~N}_{4}$ ) is negatively charged at neutral $\mathrm{pH}$, and protein layers are often negatively charged as well, the electrostatic forces are frequently repulsive. In biological systems, van der Waals interactions do not depend on the ionic strength, they decay rapidly and are always attractive. The DLVO (Derjaguin, Landau, Verwey, Overbeek) theory describes these forces quantitatively and allows the interactions between a spherical tip and a planar sample to be modeled, providing clues to optimize the recording conditions [6].

While suppliers specify tip radii of $10-50 \mathrm{~nm}$, topographs of flat biological surfaces that exhibit a resolution of $1 \mathrm{~nm}$ have been acquired routinely [4][20-22]. Therefore, the tips employed most likely had a single nm-sized asperity that protruded sufficiently to contour the finest surface structures. Such a small asperity exerts a prohibitively high pressure on the underlying structure, inducing its deformation. However, electrolytes can be used to adjust the tip-sample interactions, provided that the electrostatic forces are repulsive. The tip then surfs on a cushion of electrostatic repulsion while the small asperity is in contact with the sample [6]. Fig. 3B illustrates this situation, and it shows the changes of repulsive forces between the stylus and the sample resulting from changes of the ionic strength.

Operating commercial AFMs under such optimal recording conditions, the surfaces of biomolecules are contoured routinely at a lateral resolution better than $1 \mathrm{~nm}$ and a vertical resolution around $0.1 \mathrm{~nm}$. Fig. 4 shows some examples that document the amazing quality of topographs acquired with the AFM. Fig. 4A displays bacterial light-harvesting complexes reconstituted in a lipid bilayer [5]. These ring-shaped complexes exist in two types, the LH2 complexes that consist of nine $\alpha / \beta$ heterodimers, and the LH1 complexes consisting of $16 \alpha / \beta$ heterodimers. The latter rings are larger and accommodate the reaction center. Both rings are buried in the bilayer and contain two chromophores per $\alpha / \beta$ heterodimer that either collect the light or act as couplers, funneling the photon energy to the reaction center. The example shown documents the possibility to image macromolecular complexes within a native membrane and to study their interactions. Fig. 4B documents how the conformational space of a membrane protein surface can be sampled with the AFM [23]. In this case, a native 2D crystal composed of bacteriorhodopsin (bR) trimers and lipids has been studied. The topograph shows the conformation of the cytoplasmic surface of $b R$ in its most native state. To record this image, the force applied to the stylus was approximately $50 \mathrm{pN}$, preventing a force induced conformational change of the loop connecting helices $\mathrm{E}$ and $\mathrm{F}$. This loop is deformed when the force is increased to $>100 \mathrm{pN}$, thereby changing the conformation of the bR surface. Interestingly, the variation of this region among different atomic models from X-ray crystallography is pronounced: the EF loop is involved in the contacts leading to the 3D crystals and its conformation is dictated by the 3D packing arrangement of the bR molecules. Although all the bR trimers are identical in the $2 \mathrm{D}$ crystal dis- played in Fig. 4B, their surface structure varies significantly. These changes are related to the flexibility of the bR surface rather than any noise introduced by the AFM. The probability $\mathrm{p}_{\mathrm{l}}(\mathrm{x}, \mathrm{y})$ to find a certain loop at a certain position $(\mathrm{x}, \mathrm{y})$ can be determined by mapping the corresponding peak positions of all individual $\mathrm{bR}$ trimers. This map is readily converted to a free energy landscape $\mathrm{F}_{1}$ using Boltzmann's law:

$$
\mathrm{F}_{\mathrm{d}}(\mathrm{x}, \mathrm{y})=-\mathrm{kT} \ln \left\{\mathrm{p}_{\mathrm{l}}(\mathrm{x}, \mathrm{y})\right\}
$$

Forces need to be minimized to obtain high-resolution images, whether the AFM is operated in the contact mode or the tapping mode. However, the stylus may be used as a nanoscalpel to disrupt supramolecular assemblies [22, 25]. In this case, the force applied to the tip is increased to 1-10 $\mathrm{nN}$, depending on the damage to be achieved. Quite small forces (typically $1 \mathrm{nN}$ ) are sufficient to separate stacked layers of membranes or 2D crystals [22, 26] (Fig. 5A). Even smaller forces and repeated scanning at high magnification suffice to push away extrinsic proteins that are specifically complexed to an integral membrane protein [27].

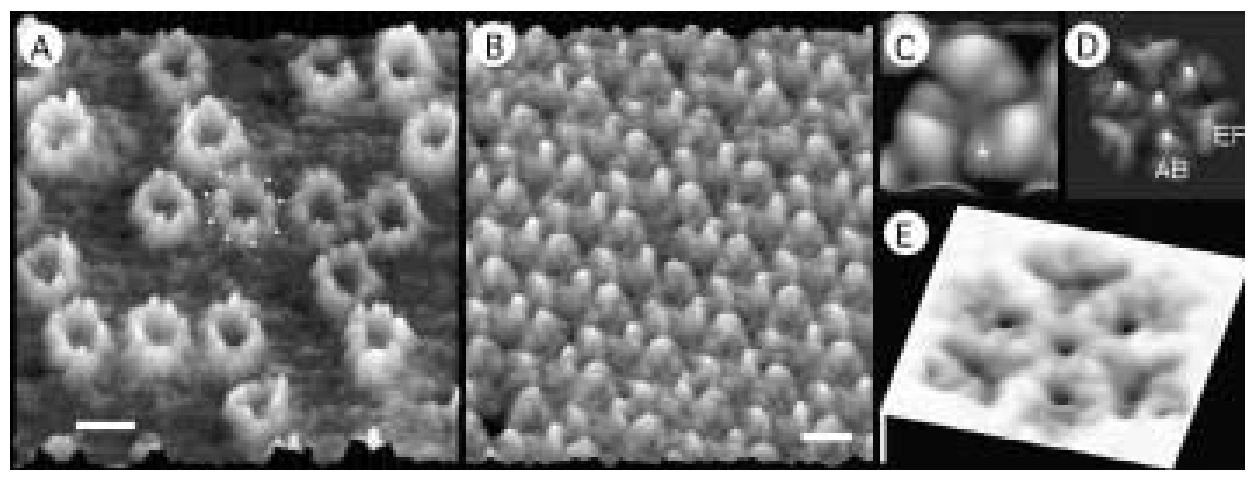

Fig. 4. AFM imaging. A) Reconstituted light-harvesting complexes LH2 of Rubrivivax gelatinosum recorded with a commercial AFM in buffer solution. LH2 is a cylindrical structure that spans the membrane and has a diameter of $5 \mathrm{~nm}$. LH2 is assembled from nine $\alpha / \beta$ heterodimers that protrude by $1.4 \mathrm{~nm}$ from the membrane surface [5]. The nine ends of the heterodimers are particularly distinct in the ring marked with dots. This topograph suggests that the AFM is suitable for imaging native membranes at high resolution. B-E) Sampling of the conformational space of a protein surface with the AFM [23]. Panel B) displays the cytosolic surface of a purple membrane, which consists of regularly packed bacteriorhodopsin (bR) trimers and lipids. The major protrusions at the periphery of the trimers (see C) protrude $0.8 \mathrm{~nm}$ out of the membrane and represent the loop connecting helices $\mathrm{E}$ and $\mathrm{F}$. Smaller protrusions reflect the shorter loop connecting helices A and B. At a closer look into panel B) significant variability of the trimer topography is seen. While the average in C) reflects the prominent features of the cytosolic bR surface, the map in panel $\mathrm{D}$ ) displays the probabilities of finding maxima related to protruding features at a given position. The EF-loops exhibiting significant flexibility are more delocalized than the AB-loops that occupy a defined position (marked with an asterisk in $\mathrm{C}$ ). The signal about the three-fold axis results from a lipid molecule protruding out by $0.1 \mathrm{~nm}$, which is only occasionally visible in the raw data, but if present precisely localized in the center of the trimer. Therefore, a strong signal emerges in the probability map (D), whereas no signal is present in the average (C). Precise localization of a surface feature suggests the corresponding structure to be stabilized in a deeper potential well than a floppy feature, which may exhibit a pronounced thermal motion. The corresponding energy landscape is shown in E) (see text). The scale bars in A) and B) represent $5 \mathrm{~nm}$ and the full gray scale is $2 \mathrm{~nm}$ in A) and $1 \mathrm{~nm}$ in B). C)-E) have a side length of $5 \mathrm{~nm}$. 


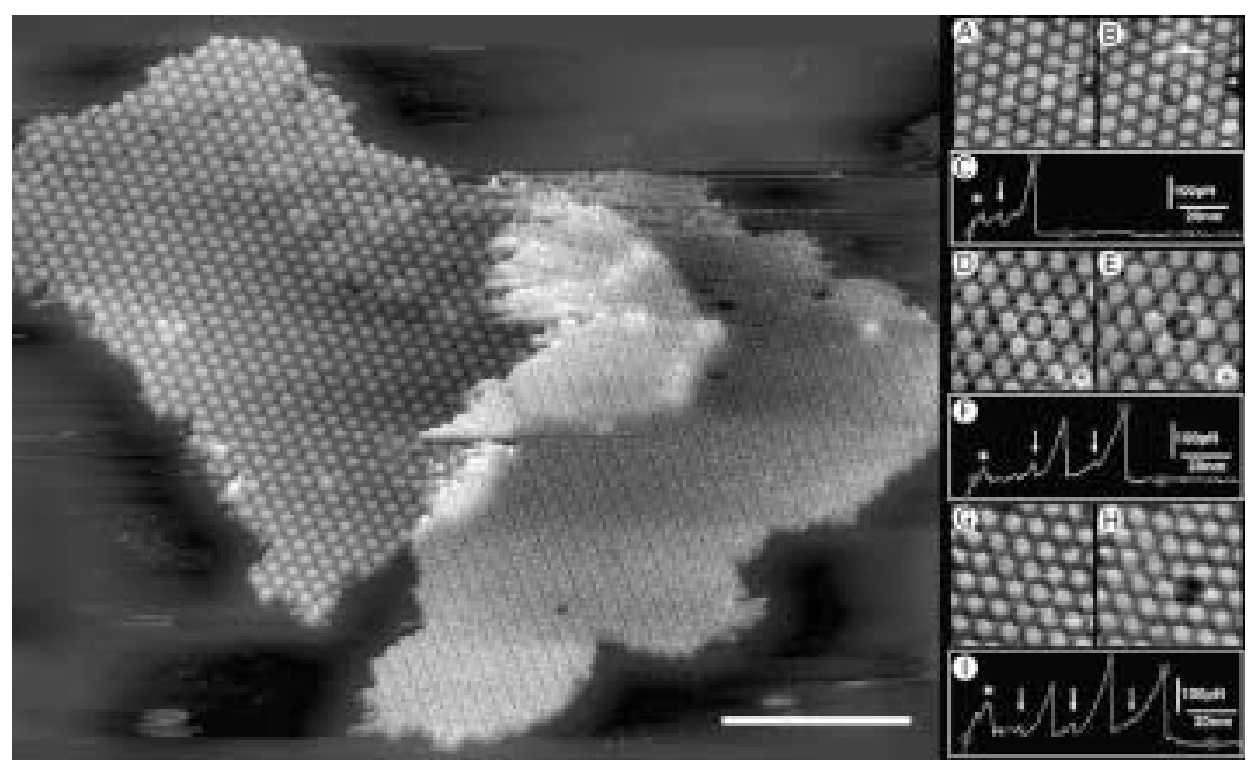

Fig. 5. AFM imaging. Manipulating supramolecular assemblies and single molecules with the AFM. Bacterial surface layers (S-layers) are regular protein networks that protect archaea and bacteria from hostile environments. The left panel displays stacked S-layers isolated from the Corynebacterium glutanicum [26]. As result of precise hydrophobic interactions these S-layers stack in register, burying the corrugated surface (left layer). To unveil this surface, the top surface of the sandwich (here on the right) can be pushed away by the stylus. A-I) Unzipping of single and multiple proteins building this S-layer is illustrated by three cases. The surface before and the damaged surface after the molecular manipulation reveal the vacancy produced by the molecular unzipping process (A \& B, D \& E, G \& H). Asterisks indicate features to identify the scanned area. The corresponding force-extension curves display the energetics of the unfolding process. Each event consists of a weak (arrow) and a strong force peak and corresponds to the extraction of a protein dimer from the hexameric unit building the hexagonal S-layer. Because the structure of the hexamer is not known, the force curves cannot be fully interpreted [25]. However, the reproducibility of these peaks suggests that it will ultimately be possible to extract structural knowledge from such data. Note that the first peaks $\left(^{*}\right)$ in the forceextension curves are the most variable, because non-specific sample-tip interactions are involved. The scale bar represents $200 \mathrm{~nm}$ and the subframes have a side length of $100 \mathrm{~nm}$. The grey scale corresponds to $5 \mathrm{~nm}$.

\section{Single Molecule Force Spectroscopy}

Today, instruments offer a high spatial resolution and force sensitivity down to the piconewton range. They allow the forces of single molecular interactions to be measured [28-31]. For single molecule force spectroscopy using the AFM, molecules must be tethered to both the support and the tip in some way. Whilst a variety of chemical surface activation techniques have been used, large 2D assemblies such as bacterial S-layers or reconstituted membranes are simply physisorbed to mica (Fig. 5, left panel). The AFM stylus is then attached to an individual protein of such an assembly by pressing the stylus down with a force of $\sim 1 \mathrm{nN}$ for approximately $1 \mathrm{~s}$, which induces the denaturation of some protruding domain that is thus stuck to the silicon nitride tip. Specifically designed mutant proteins that bear a cystein on a surface-exposed domain allow specific attachment of the protein to a gold-coated stylus. Alternatively, a ligand may be covalently immobilized on the stylus through a suitable spacer, while the receptor is tethered to the support.
When approaching the tip to the support, a bond may form between ligand and receptor. In all cases, the change in cantilever deflection upon tip retraction is then recorded, yielding a force-extension curve (Fig. 5 right panel) [26]. By scanning the sample before (Fig. 5 A,D,G) and after (Fig. 5 $\mathrm{B}, \mathrm{E}, \mathrm{H})$ such molecular 'unzipping' events, the structural damage can be directly assessed and correlated with the respective force-extension curve (Fig. 5 C,F,I).

In addition, the AFM offers the possibility of unbinding biomolecular bonds (e.g. ligand/receptor). Because the unbinding of biomolecular bonds is a dynamic process, the acquisition of force-extension curves at various extension rates in these experiments is also referred to as dynamic force spectroscopy (DFS). It has long been known that only molecules with an excess of energy over the average energy of the population can participate in chemical reactions. Accordingly, reactions between ligands and receptors follow pathways (in a virtual energy landscape) that involve the formation of some type of high-energy tran- sition states whose accessibility along a reaction coordinate ultimately controls the reaction rate. But because quantitative measurements have only been possible on large molecular ensembles, only macroscopic thermodynamical quantities could be determined, e.g. the free energy of complex formation and/or dissociation. From single molecule measurements, the energy landscape of a single molecular interaction can be mapped, giving a detailed insight into the reaction pathway of single molecular processes. Detailed thermodynamical models describing the rupture of a single bond are described in [32-34].

DFS measurements can be performed with an unmodified commercial AFM (see Fig. 5 and 6), or using external data acquisition and data output capabilities that enhance sensitivity and flexibility of the instrument. To achieve reproducible measurements, the spring constants of all cantilevers used must be calibrated. The thermal fluctuation method [35] is currently the most convenient method and gives an absolute uncertainty of $20 \%$. For the temper- 
ature measurements presented below, the temperature was controlled using a homebuilt cell in which the buffer solution that immersed both the probe surface and the AFM cantilever was in contact with a Peltier element, driven with a constant current source. Measurements at different points of the cell showed deviations of less than $2{ }^{\circ} \mathrm{C}$. Preparation and immobilization of all oligonucleotides was carried out using the protocol described in [31][36].

Unbinding events are caused by thermal fluctuations rather than by mechanical instability. Therefore unbinding forces show a distribution whose width $\sigma$ is mainly determined by the force scale $F^{0}$, i.e. $\sigma=F^{0}(\Delta x)$.

When approaching the tip to the surface, many non-specific attachments may occur, even in the presence of treated surfaces or pure polymer samples. Therefore, it is imperative to test the specificity of the interaction (see inset Fig. 6).

Unspecific interactions can be minimized using linkers (e.g. poly(ethylene) glycol (PEG) linkers) that shift the region where unbinding takes place away from the surface. Finally, to quantify the most probable value for the unbinding force of a single complex, one has to work under conditions in which the probability that two or more complexes are attached to the tip is low.

These conditions are fulfilled for a low concentration and when the linkers have a length that is comparable to the diameter of the AFM-tip (about 10-50 nm). In this case, it is very unlikely that two or more linkers are extended to the same length when stretched. Nevertheless, subsequent rupture events may be found. But still, the last rupture event will occur for an applied force equal to $F^{*}$ (see Eqn. 2).

DFS measurements have been performed on complementary DNA strands $(10,20$, and 30 base pairs (bp)) that were pulled apart at their opposite 5'-ends. The base sequences of the oligonucleotides were designed to favor the binding to its complementary oligonucleotides in the ground state with respect to intermediate duplexes in which the strand is shifted relative to its complement. We have chosen the oligomer a (5'-G-G-C-T-C-C-C-T-T-C-TA-C-C-A-C-T-G-A-C-A-T-C-G-C-A-A-CG-G-3'), which contains 30 bases and in which every three base motive occurs only once in the sequence. For this sequence, self-complementarities are avoided because the complement of each three-base motive is not contained in the sequence. a was tested against its complement b (30 bp) and against truncated components $\mathbf{c}$ (20 bp) and $\mathbf{d}$ (10 bp), respectively.

As expected, a $F^{*}$ versus $\ln (v)$ plot shows a linear behavior for each duplex (Eqn. 2, see Fig. 6).

For each duplex, the distance $\Delta x$ from the ground state to the energy barrier and the thermal off rate $k_{\text {off }}$ were determined according to Eqn. 2.

$$
F^{*}=F^{0} \ln \left(r / F^{0} k_{o f f}\right)
$$

The $\Delta x$ distance was found to follow the linear relation: $\Delta x=[(0.7 \pm 0.3)+(0.07 \pm$ $0.03) \times n] \mathrm{nm}$, where $n$ is the number of base pairs. This increase of $\Delta x$ with $n$ clearly indicates cooperativity in the unbinding process. Measurements of $k_{\text {off }}$ can be described by: $k_{\text {off }} 10^{\alpha-\beta \mathrm{n}^{-1}}$, where $\alpha=3 \pm 1$ and $\beta=0.5 \pm 0.1$. The obtained $k_{\text {off }}$ values are in good agreement with thermodynamical data [37]. Let us finally point out that an exponential decrease of the thermal off-rate with the number of base pairs is expected because of the increase of the activation energy for dissociation.

In this section, temperature dependent DFS measurements are briefly discussed. The sequence e (5'-T-A-T-T-A-A-T-A-T-CA-A-G-T-T-G-3') [38] attached to the tip and its complement $\mathbf{f}$ was immobilized on the surface. As previously, PEG linkers were used and DNA strands were pulled apart at their opposite 5'-ends. The specificity of the interaction was comparable to the one obtained in base-pair dependent measurements (Fig. 6). One striking difference of these energy landscape measurements is visible when the temperature is varied. The slope of the $F^{*}$ versus $\ln (r)$ plots changes as a function of temperature, which

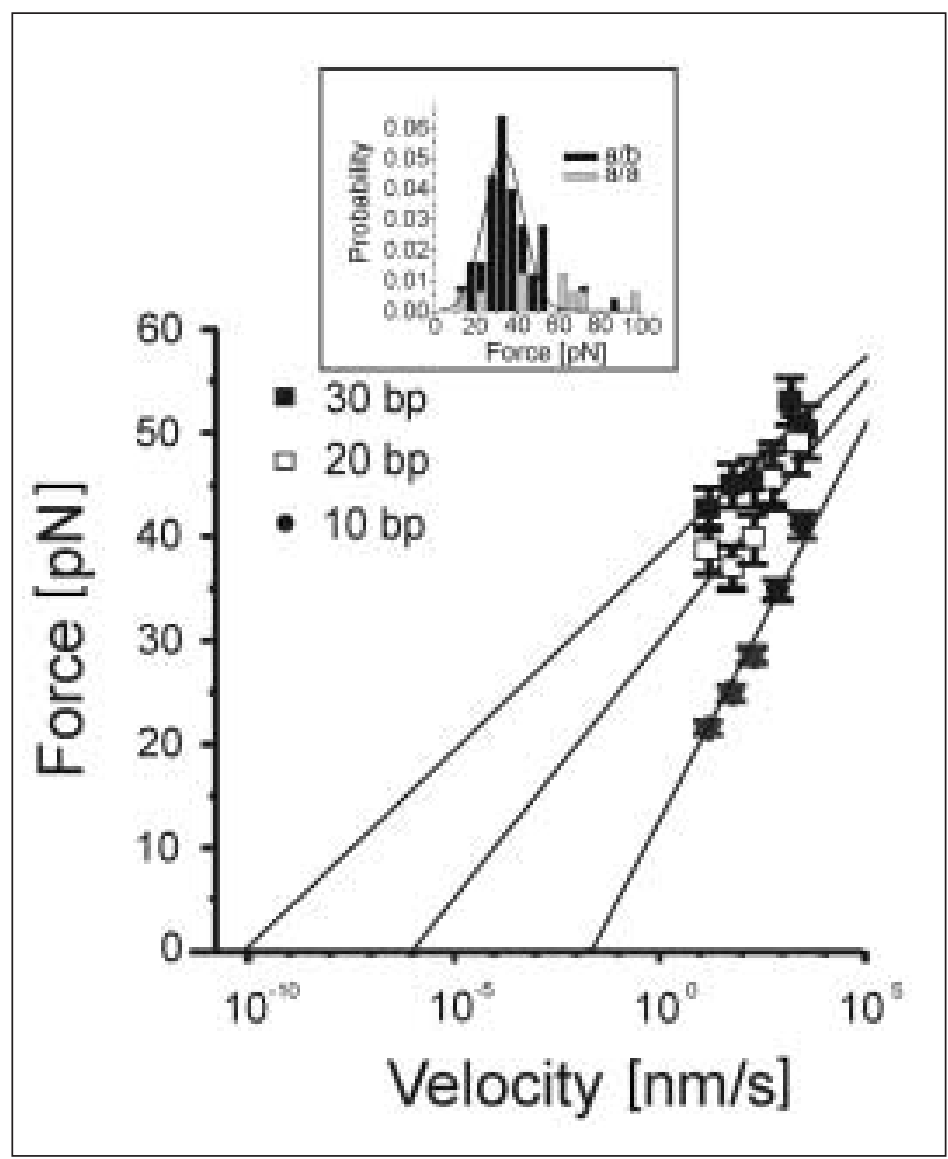

Fig. 6. Dynamic force spectroscopy. Rupturing dsDNA molecules: Velocity dependence of the most probable unbinding force. Back squares (a-tip/b-surface, 30 bp), empty squares (a-tip/c-surface, 20 bp), circles (a-tip/d-surface, $10 \mathrm{bp}$ ). From a linear fit, both the forcescales $F^{O}=k_{B} T / \Delta x$ and thermal off-rates can be determined. Inset specificity of the rupturing force: A typical probability distribution for the rupture force (about 500 approach/retract cycles, retract velocity $100 \mathrm{~nm} / \mathrm{s}$ ). For this experiment, an oligomer a (see text) was attached to the tip of the AFM-cantilever and its complement $b$ was immobilized on the surface (complements were pulling apart at their opposite 5'-ends). Gray rectangles (a against a), black rectangles (a against $b$ ). To minimize unspecific interactions (e.g. a against $a$ ) and multiple unbinding events, 30-nm long PEG linkers were attached to the $5^{\prime}$-ends. Note that the scale-force $F^{0}$ can be in principle determined from the width of the distribution. 
indicates a strong temperature dependence of $\Delta x$. This result emphasizes the fact that for the DNA-duplex, the energy landscape is much more complicated than that of ligand-receptor bonds.

Our measurements confirm that the most probable scale for unbinding forces is the logarithm of the loading rate. From this dependence, both the natural thermal offrate for dissociation $k_{\text {off }}$ and the bond length $\Delta x$ along the reaction coordinate were determined. Our measured $k_{\text {off }}$ values are in agreement with bulk temperature measurements indicating the validity of our measurements. The base pairs dependent measurements indicate that unbinding of DNA strands is a cooperative process. Temperature dependent measurements evidence for a decrease of $\Delta x$ as the temperature increases [31]. This behavior, which is not expected in the case of one-dimensional energy landscape with a sharp energy barrier, indicates the role of entropic contributions when unbinding DNA and unfolding RNA or proteins.

\section{Experiments Using Optical Tweezers}

Force extension curves may also be acquired with an optical tweezers setup [39][40]. In addition, single molecule force measurements can be combined with distance measurements using fluorescence resonance energy transfer as shown in Fig. 2 (FRET) [11]. Certainly, one of the most impressive applications of OTs is the study of molecular motors on a single molecule level. These molecular motors can be linear motors (Kinesin, Myosin) [39][40], DNA/RNA polymerase enzymes [41][42] or DNA packaging viruses (bacteriophage ф29) [43].

Kinesin and Myosin are two ATPase motor proteins. Kinesin, which is used for organelle transport or chromosome segregation, moves along microtubules. In contrast, Myosin interacts with actin filaments and is used not only for muscle contraction but also is involved in many forms of cell movement. For these studies, OTs are used to interact single Kinesin or Myosin in vitro with either a microtubule or an actin filament. These experiments have revealed how much ATP has to be hydrolyzed and the forces generated at each step, demonstrating possible mechanisms involved in the movement.

Other experiments investigated the function of motor enzymes used in DNA transcription or DNA polymerization. In this case a single DNA molecule is tethered between two beads, and the rate of transcription or polymerization can be followed in real time by applying a constant tension (force feedback) and allowing the distance between the beads to change accordingly [41][42]. Such studies have direct implications for the mechanism of gene regulation or force-induced exonuclase activity. In a recent study Smith and colleagues measured the forces involved in the packaging of DNA into bacteriophage $\phi 29$ heads [43]. A schematic picture of these experiments is given in Fig. 7 (top). The key elements of such an experiment are the native immobi-

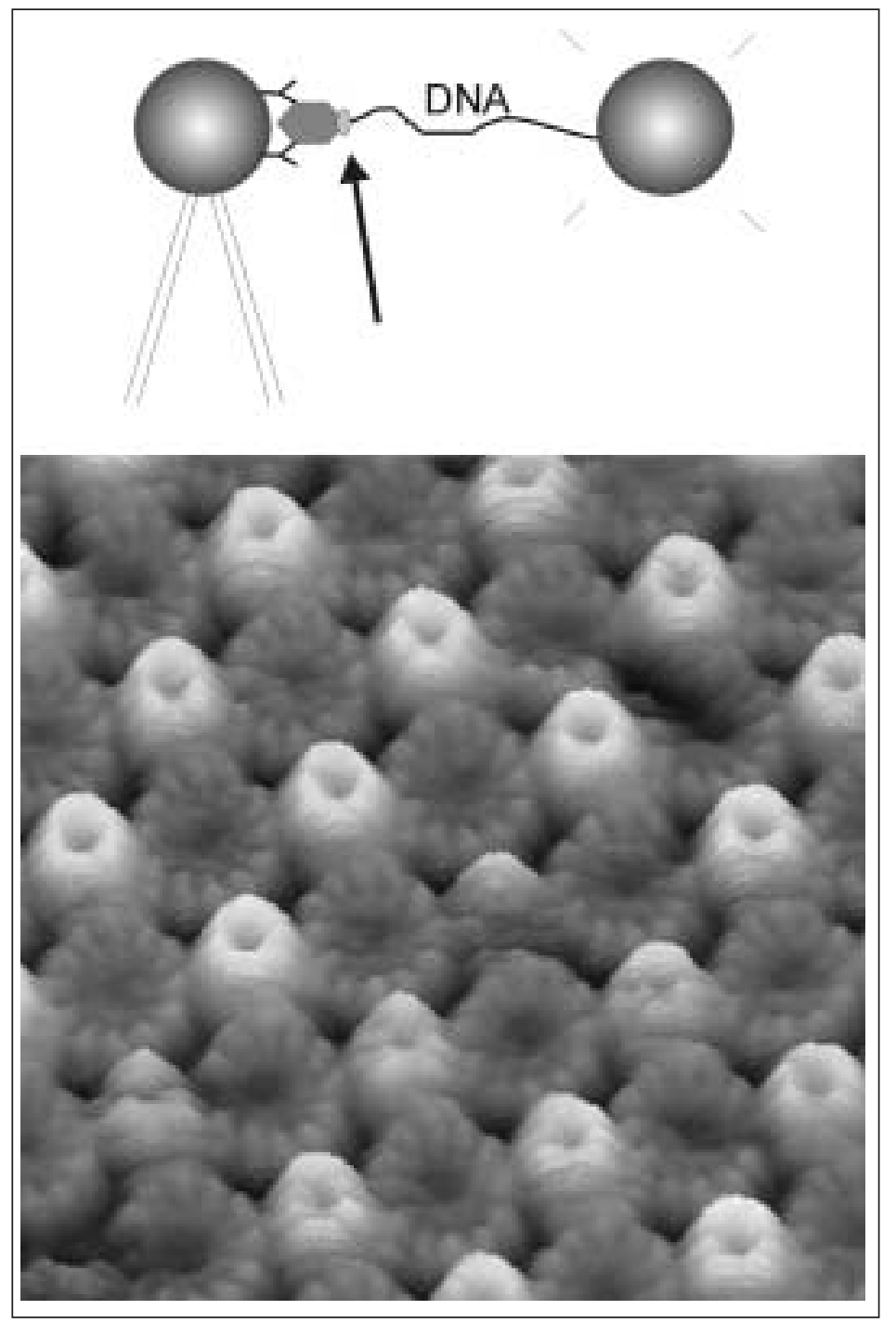

Fig. 7. OTs experiments: Possible experimental setups used for single molecule observation of molecular motors. Top: A bead coated with a bacteriophage head (grey) is held by suction on top of a micropipette. This head is interacting with a DNA molecule attached on a second bead trapped by an optical tweezers. DNA is recognized by molecular motor on the phage head responsible for DNA packaging in to the head. The motor spools the single DNA molecule into the head and is exerting a rather high force on the bead sitting in the trap. Bottom: AFM image of a two-dimensional ø29 connector crystal recorded in buffer solution [45]. In presence of $1 \mathrm{M} \mathrm{KCl}$ the planar sheets adsorbed smoothly onto the freshly cleaved mica surface. The average height of the crystals directly attached to mica was $8.3 \pm 0.6$ $\mathrm{nm}$. Because connectors were packed in opposite orientations, both sides are visible: one exhibiting a cylindrical protrusion, and the other showing a wheel-like structure with twelve radial petals. The cylinder connects to the phage tail, whereas the wheel connects to the icosahedral phage head via a set of molecular motors. The cylindrical were $16.5 \mathrm{~nm}$ apart. Imaging conditions: buffer solution $(0.5 \mathrm{M}$ $\mathrm{KCl}, 20 \mathrm{mM}$ Tris-HCl, pH 8.4). Applied force was about $500 \mathrm{pN}$. Scan frequency was $3.2 \mathrm{~Hz}$ at a frame size of $1.4 \mu \mathrm{m}$. Scale bars of a) and b) were $2.5 \mu \mathrm{m}$ and $100 \mathrm{~nm}$, respectively. Full gray level range of a) and b) were $50 \mathrm{~nm}$ and $4 \mathrm{~nm}$, respectively. 
lization of the bacteriophage head onto the surface of one bead and the attachment of the DNA molecule [44] to the second microsphere. A high-resolution AFM image of the bacteriophage $\phi 29$ packaging motor protein complex is shown in Fig. 7 [45].

Due to the high force sensitivity, OTs have been used to study (i) mechanical properties of DNA [46] (ii) protein or RNA unfolding [47] (iii) the polymerization of individual RecA-DNA filaments [48]. Again, these experiments provided new insights in biochemical processes on a single molecule and are of great relevance to biology. In a recent publication we were able to measure directly the kinetics of binding of small ligands to dsDNA using optical tweezers experiments [49]. By recording a force versus distance experiment we are able to extract the mechanical parameters of the modified dsDNA molecule directly (Fig. 8). The parameters obtained indicate the way of binding: if intercalation occurs then the contour length is affected. Additionally we can determine the occupancy of the ligand on the DNA from such measurements and see how the native mechanics of the molecule is altered. If compounds bind to dsDNA which are not intercalating then the binding is directly revealed in the way the modified dsDNA is going through its overstretch transition. Such experiments can give direct insight into the binding of small ligands to DNA and can be of great importance for drug screening.

\section{Perspectives}

Progress in instrumentation and sample preparation methods have opened avenues to image, manipulate and assess the nanomechanics of single molecules. Such novel experimental possibilities find a wide range of applications in the study of structure and function of biomolecules, the building blocks of life. While the amazing S/N-ratio and resolution of the AFM allows the conformational states of single protein surfaces to be probed at a lateral resolution of $0.4-1 \mathrm{~nm}$ lateral and $0.1-0.2 \mathrm{~nm}$ vertical resolution, the force sensitivity ( $>20 \mathrm{pN}$ ) of this instrument also opens the door to measure the forces required to unfold a protein. Combining single molecule imaging and force spectroscopy gives an unprecedented insight into the nature of intra- and intermolecular interactions in and between biomolecules.

Optical tweezers enhance the force sensitivity compared to the AFM by an order of magnitude. Therefore, OTs are suited to monitor molecular motors that generate forces in a range of 0.3 to $50 \mathrm{pN}$ while at work. Using light, particles of some nm up to some $\mu \mathrm{m}$ in diameter can be trapped and manipulated. Simultaneously, fluorescence techniques allow small distance changes to be measured, allowing a full analysis of the nanomechanical features of a single molecule.

Using DFS measurements, the energy landscape of molecular bonds can be mapped. Moreover, relevant parameters such as the location and height of the barriers and the thermal off-rates can be determined. Since the limited range of loading rates available in an AFM experiment does not allow one to map the whole energy landscape, such experiments should be combined in the future with other DFS setups such as bio-membrane force probe or optical tweezers instruments. An additional solution is to apply small cantilevers, which allow faster pulling and exhibit less thermal noise; so smaller unbinding forces can be detected. These small cantilevers are still experimental [50] and great efforts are being made to commercialize them in the future. These developments will also ask for instrument development so it will take a few years before they are widely used. One could envision that the dynamic force spectroscopy will be applied in the future to assess the binding affinity of biomolecules on bio-arrays and experimentalists are likely to move in this direction.

\section{Acknowledgement}

Financial support of the NCCR 'Nanoscale Science' the Swiss National Science Foundation and the ELTEM Regio Project Nanotechnology is gratefully acknowledged.

Received: July 25, 2002

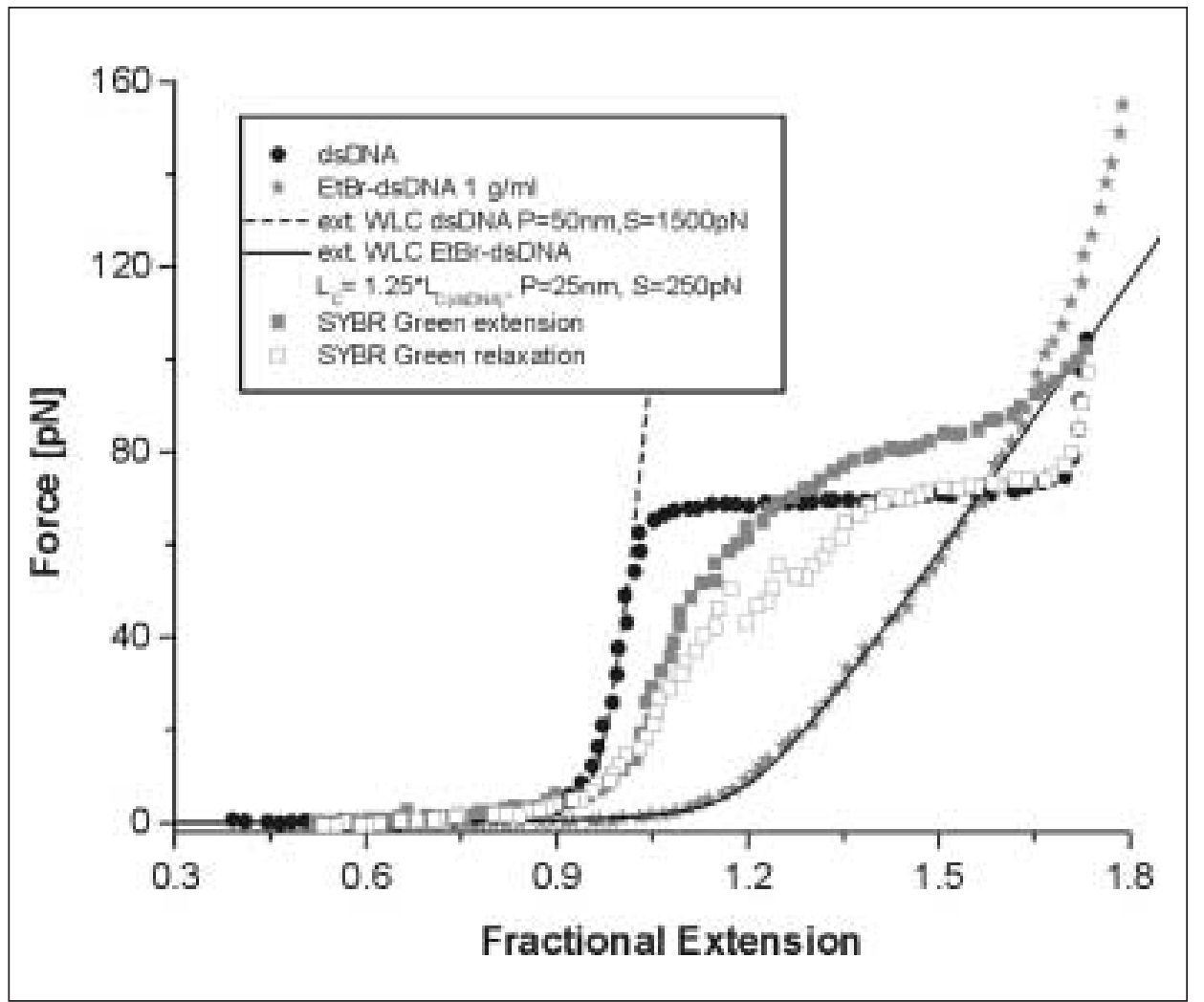

Fig. 8. DNA mechanics: Typical force versus fractional extension $x / L$ curves obtained for bare dsDNA (circles); and EtBr (stars). SYBR ${ }^{\circledR}$ Green I dsDNA (squares, full squares extension, hollow squares relaxation), Also shown is the fit to the data using an extensible worm like chain model (dashed lines). From this fit; relevant enthalpic parameters (stretch modulus) are obtained. 
[1] G. Binnig, C.F. Quate, C. Gerber, Phys. Rev. Lett. 1986, 56, 930-933.

[2] J.X. Mou, J. Yang, Z.F. Shao, J. Mol. Biol. 1995, 248, 507-512.

[3] D.J. Müller, M. Amrein, A. Engel, J. Struct. Biol. 1997, 119, 172-188.

[4] D.M. Czajkowsky, S. Sheng, Z. Shao, J. Mol. Biol. 1998, 276, 325-330.

[5] S. Scheuring, F. Reiss-Husson, A. Engel, J.L. Rigaud, J.L. Ranck, Embo. J. 2001, 20, 3029-3035.

[6] D.J. Müller, D. Fotiadis, S. Scheuring, S.A. Müller, A. Engel, Biophys. J. 1999, 76, 1101-1111.

[7] C. Möller, M. Allen, V. Elings, A. Engel, D.J. Müller, Biophys. J. 1999, 77, 1050-1058.

[8] P.K. Hansma, J.P. Cleveland, M. Radmacher, D.A. Walters, P.E. Hillner, M. Bezanilla, M. Fritz, D. Vie, H.G. Hansma, C.B. Prater, et al. Appl. Phys. Lett. 1994, 64, 1738-1740.

[9] W. Han, S.M. Lindsay, M. Dlakic, R.E. Harrington. Nature 1997, 386, 563.

[10] M.B. Viani, T.E. Schaffer, G.T. Paloczi, L.I. Pietrasanta, B.L. Smith, J.B. Thompson, M. Richter, M. Rief, H.E. Gaub, K.W. Plaxco, et al. Rev. Sci. Instr. 1999, 70, 4300-4303.

[11] W. Grange, S. Husale, H.-.J. Güntherodt, M. Hegner, Rev. Sci. Instr. 2002, 73, 2308-2316.

[12] A. Ashkin, Phys. Rev. Lett. 1970, 24, 156.

[13] A. Ashkin, J.M. Dziedzig, J.E. Bjorkholm, S. Chu, Opt. Lett. 1986, 11, 288-290.

[14] A. Ashkin, J.M. Dziedzig, T. Yamane, $\mathrm{Na}$ ture 1987, 330, 769-771.

[15] A. Ashkin, J.M. Dziedzig, Science 1987, 235, 1517-1520.

[16] K. Svoboda, S.M. Block, Ann. Rev. Biophys. Biomol. Struct. 1994, 23, 247-285.

[17] G. Leitz, E. Fällmann, S. Tuck, O. Axner, Biophys. J. 2002, 82, 2224-2231.

[18] K.C. Neuman, E.H. Chadd, G.F. Liou, K. Bergman, S.M. Block, Biophys. J. 1999, 77, 2856-2863.

[19] R.S. Afzal, E.B. Treacy, Rev. Sci. Instr. 1992, 63, 2157-2163.

[20] A. Engel, D.J. Müller, Nat. Struct. Biol. 2000, 7, 715-718.

[21] D.J. Müller, A. Engel, J. Mol. Biol. 1999, 285, 1347-1351.

[22] F.A. Schabert, C. Henn, A. Engel, Science 1995, 268, 92-94.

[23] S. Scheuring, D. Müller, H. Stahlberg, H. Engel, A. Engel, European Biophys. J. 2002, 31, 172-178.

[24] D.J. Müller, J.B. Heymann, F. Oesterhelt, C. Moller, H. Gaub, G. Buldt, A. Engel, Biochim. Biophys. Acta 2000, 1460, 27-38.

[25] D. Fotiadis, S. Scheuring, S.A. Müller, A. Engel, D.J. Müller, Micron 2002, 33, 385-397.

[26] S. Scheuring, H. Stahlberg, M. Chami, C. Houssin, J.L. Rigaud, A. Engel, Mol. Microbiol. 2002, 44, 675-684.

[27] D. Fotiadis, D.J. Müller, G. Tsiotis, L. Hasler, P. Tittmann, T. Mini, P. Jenö, H. Gross, A. Engel, J. Mol. Biol. 1998, 283, 83-94.
[28] S. Allen, X.Y. Chen, J. Davies, M.C. Davies, A.C. Dawkes, J.C. Edwards, C.J. Roberts, J. Sefton, S.J.B. Tendler, P.M. Williams, Biochemistry 1997, 36 7457-7463.

[29] E. Evans, A. Leung, D. Hammer, S. Simon, Proc. Natl. Acad. Sci. USA 2001, 98 , 3784-3789.

[30] V.T. Moy, E.L. Florin, H.E. Gaub, Science 1994, 266, 257-259.

[31] I. Schumakovitch, W. Grange, T. Strunz, P. Bertoncini, H.-J. Güntherodt, M. Hegner, Biophys. J. 2002, 82, 517-521.

[32] E. Evans, Ann. Rev. Biophys. Biomol. Struct. 2001, 30, 105-128.

[33] E. Galligan, C.J. Roberts, M.C. Davies, S.J.B. Tendler, P.M. Williams, J. Chem. Phys. 2001, 114, 3208-3214.

[34] B. Heymann, H. Grubmüller, Phys. Rev. Lett. 2000, 84, 6126-6129.

[35] J.L. Hutter, J. Bechhöfer, Rev. Sci. Instr. 1993, 64, 3342-3342.

[36] T. Strunz, K. Oroszlan, R. Schäfer, H.-J. Güntherodt, Proc. Natl. Acad. Sci. USA 1999, 96, 11277-11282.

[37] D. Pörscke, M. Eigen, J. Mol. Biol. 1971, $62,361$.

[38] N. Tibanyenda, S.H. Debruin, C.A.G. Haasnoot, G.A. Vandermarel, J.H. Vanboo, C.W. Hilbers, Eur. J. Biochem. 1984, 139, 19-27.

[39] C. Bustamante, J.C. Macosko, G.J.L. Wuite, Nature Rev. Mol. Cell Biol. 2000, 1 , 130-136.

[40] A.D. Mehta, M. Rief, J.A. Spudich, D.A. Smith, R.M. Simmons, Science 1999, 283, 1689-1695.

[41] R.J. Davenport, G.J.L. Wuite, R. Landick, C. Bustamante, Science 2000, 287, 2497-2500.

[42] G.J.L. Wuite, S.B. Smith, M. Young, D. Keller, C. Bustamante, Nature 2000, 404, 103-106.

[43] D.E. Smith, S.J. Tans, S.B. Smith, S. Grimes, D.L. Anderson, C. Bustamante, Nature 2001, 413, 748-752.

[44] M. Hegner, Single Mol. 2000, 1, 139-144.

[45] D.J. Müller, A. Engel, J.L. Carrascosa, M. Velez, Embo. J. 1997, 16, 2547-2553.

[46] M.C. Williams, I. Rouzina, Curr. Op. Struct. Biol. 2002, 12, 330-336.

[47] J. Liphardt, S. Dumont, S.B. Smith, I. Tinoco, C. Bustamante, Science 2002, 296, 1832-1835.

[48] M. Hegner, S.B. Smith, C. Bustamante, Proc. Natl. Acad. Sci. USA 1999, 96, 10109-10114.

[49] S. Husale, W. Grange, M. Hegner, Single Mol. 2002, 3, 91-96.

[50] M.B. Viani, T.E. Schäfer, A. Chand, M. Rief, H. Gaub, P.K. Hansma, J. Appl. Phys. 1999, 86, 2258-2262. 